\title{
Large eddy simulation and the filtered equation of a contaminant
}

\author{
F. Gallerano, L. Melilla \& G. Cannata \\ Dipartimento di Idraulica, Trasporti e Strade, Università "La Sapienza", \\ Roma, Italia
}

\begin{abstract}
A new model of LES is proposed together with a new methodology for the simulation of concentration fields of contaminant coherent with the LES methodology. In this paper a new LES model is proposed. The closure relation for the generalised SGS turbulent stress tensor: a) complies with the principle of turbulent frame indifference; b) takes into account both the anisotropy of the turbulence velocity scales and turbulence length scales; c) removes any balance assumption between the production and dissipation of SGS turbulent kinetic energy. In the proposed model: a) the closure coefficient which appears in the closure relation for the generalised SGS turbulent stress tensor is theoretically and uniquely determined without adopting Germano's dynamic procedure; b) the generalised SGS turbulent stress tensor is related exclusively to the generalised SGS turbulent kinetic energy (which is calculated by means of its balance equation) and the modified Leonard tensor. The calculation of the viscous dissipation is carried out by integrating its exact balance equation. In this paper the form invariance and frame dependence of the above mentioned equation of the viscous dissipation transport is shown. The concentration field is simulated by the spatially filtered equation of the concentration. In this equation the first-order tensor (produced by the correlation between the velocity and the concentration) is related to the gradient of the resolved concentration according to an original dynamic Germano procedure.
\end{abstract}

Keywords: concentration, contaminant, LES, turbulence.

\section{A new LES model}

In order to remove the assumption of alignment between the unresolved part of the generalised SGS turbulent stress tensor and the resolved strain rate tensor and 
to take into account the anisotropy of the unresolved scales of turbulence, the generalised SGS turbulent stress tensor is expressed in the following form

$$
\tau_{i j}=L_{i j}{ }^{m}-2 v_{i j m n} \bar{S}_{m n}
$$

in which $\bar{S}_{m n}$ is the resolved strain rate tensor and $L_{i j}^{m}$ is the modified Leonard tensor. The eddy viscosity is expressed in the above-mentioned equation by a fourth-order tensor proportional to the product of a second-order tensor, $b_{i j}$, which represents the turbulence velocity scales, and a second-order tensor, $d_{m n}$, which represents the turbulence length scales, according to the following equation:

$$
v_{i j m n}=C b_{i j} d_{m n} \text { where } b_{i j}=b_{j i}, \quad d_{m n}=d_{n m} .
$$

The expression of the eddy viscosity in terms of a fourth-order tensor enables the anisotropic character of the turbulence to be fully represented, since it does not either assume the existence of a single turbulence velocity scale and or a single turbulence length scale, as is found in models in which the viscosity is expressed as a scalar. The second-order tensor which represents the turbulence velocity scales is defined as

$$
b_{i j}=\sqrt{E} \frac{L_{i j}^{m}}{L_{k k}^{m}}
$$

where $E$ is the SGS turbulent kinetic energy. The second-order tensor $d_{m n}$ is defined as

$$
d_{m n}=\Delta_{m} \Delta_{n} /\left(\Delta_{1} \Delta_{2} \Delta_{3}\right)^{\frac{1}{3}}
$$

in which $\Delta_{i}$ is the vector of which the components are the filter dimensions in the three coordinate directions.

In this manner it is assumed that the anisotropy of the unresolved turbulence velocity scales, expressed by tensor $b_{i j}$, is equal to the anisotropy of the smallest resolved scales, associated with the modified Leonard tensor. This assumption is based on scale similarity, according to which the scales that are contiguous in the wavenumber space have strict dynamic analogies related to the energy exchange processes which occur between them.

Introducing eqn (2) and (3) into (1) gives:

$$
\tau_{i j}=(1+r) L_{i j}^{m} \quad \text { where } \quad r=-2 C d_{m n} S_{m n} \frac{\sqrt{E}}{L_{k k}^{m}}
$$

In order to close the equations governing the turbulent flows, it is necessary to determine the coefficient $C$ which appears in eqn (4.a) or else, the coefficient $r$ which appears in eqn (4.b).

In this paper the coefficient $r$ is determined without Germano's dynamic procedure, the inconsistencies of which are fundamentally linked to the fact that 
in the wall region the dimensions of the filters used in the dynamic procedure are larger than those of the largest eddies governing the energy and momentum transfer.

The coefficient $r$ is uniquely and theoretically determined by using the relation between the generalised SGS turbulent kinetic energy and the generalised SGS turbulent stress tensor. In fact, by definition, the generalised SGS turbulent kinetic energy is equal to half the trace of the generalised SGS turbulent stress tensor.

$$
\tau_{k k}=2 E=(1+r) L_{k k}^{m} \quad \text { i.e. } \quad r=\frac{2 E-L_{k k}^{m}}{L_{k k}^{m}}
$$

introducing (5) into (4) gives

$$
\tau_{i j}=\left(1+\frac{2 E-L_{k k}^{m}}{L_{k k}^{m}}\right) L_{i j}^{m}=\left(\frac{2 E}{L_{k k}^{m}}\right) L_{i j}^{m}
$$

The closure relation (6) is obtained without any assumption of local balance between the production and dissipation of generalised SGS turbulent kinetic energy and may thus be considered applicable to LES with the filter width falling into the range of wave numbers greater than the wave number corresponding to the maximum turbulent kinetic energy.

The closure relation for the generalised SGS turbulent stress tensor (6): a) complies with the principle of turbulent frame indifference given that it relates only objective tensors [1]; b) takes into account both the anisotropy of the turbulence velocity scales and turbulence length scales; c) assumes scale similarity in the definition of the second-order tensor representing the turbulent velocity scales; d) guarantees an adequate energy drain from the grid scales to the subgrid scales and guarantees backscatter; e) overcomes the inconsistencies linked to the dynamic calculation of the closure coefficient used in the modelling of the generalised SGS turbulent stress tensor.

The generalised SGS turbulent kinetic energy, $E$, is calculated by solving its balance equation, defined by the following equation:

$$
\frac{D E}{D t}=-\frac{1}{2} \frac{\partial \tau\left(u_{k}, u_{k}, u_{m}\right)}{\partial x_{m}}-\tau_{m k} \frac{\partial \bar{u}_{k}}{\partial x_{m}}-\frac{\partial \tau\left(p, u_{m}\right)}{\partial x_{m}}+v \frac{\partial^{2} E}{\partial x_{m} \partial x_{m}}+\tau\left(F_{O k}, u_{k}\right)-v \tau\left(\frac{\partial u_{k}}{\partial x_{m}}, \frac{\partial u_{k}}{\partial x_{m}}\right)
$$

Equation (7) is form-invariant under Euclidean transformations of the frame and frame-indifferent [5]. The last term on the right of (7) is defined SGS viscous dissipation:

$$
\varepsilon=v \tau\left(\frac{\partial u_{i}}{\partial x_{j}}, \frac{\partial u_{i}}{\partial x_{j}}\right)
$$

In the proposed LES model a further balance equation is introduced for the subgrid viscous dissipation $\varepsilon$. This equation, expressed in terms of the generalized central moments, takes the form: 


$$
\begin{aligned}
& \frac{\partial \varepsilon}{\partial t}+\frac{\partial \bar{u}_{k} \varepsilon}{\partial x_{k}}-v \frac{\partial^{2} \varepsilon}{\partial x_{k} \partial x_{k}}+v \frac{\partial}{\partial x_{k}} \tau\left(u_{k}, \frac{\partial u_{i}}{\partial x_{j}}, \frac{\partial u_{i}}{\partial x_{j}}\right)+2 v \frac{\partial}{\partial x_{k}}\left(\frac{\partial \bar{u}_{i}}{\partial x_{j}} \tau\left(u_{k}, \frac{\partial u_{i}}{\partial x_{j}}\right)\right) \\
& +2 v \frac{\partial}{\partial x_{k}} \tau\left(\frac{\partial u_{k}}{\partial x_{i}}, \frac{\partial p}{\partial x_{i}}\right)-2 v \frac{\partial}{\partial x_{k}}\left(\frac{\partial \bar{u}_{i}}{\partial x_{j}} \frac{\partial \tau_{i k}}{\partial x_{j}}\right)+2 v \tau\left(\frac{\partial u_{i}}{\partial x_{k}}, \frac{\partial u_{k}}{\partial x_{j}}, \frac{\partial u_{i}}{\partial x_{j}}\right) \\
& +2 v \frac{\partial \bar{u}_{k}}{\partial x_{j}} \tau\left(\frac{\partial u_{i}}{\partial x_{k}}, \frac{\partial u_{i}}{\partial x_{j}}\right)+2 v \frac{\partial \bar{u}_{i}}{\partial x_{k}} \tau\left(\frac{\partial u_{k}}{\partial x_{j}}, \frac{\partial u_{i}}{\partial x_{j}}\right)+2 v \frac{\partial \bar{u}_{i}}{\partial x_{j}} \tau\left(\frac{\partial u_{i}}{\partial x_{k}}, \frac{\partial u_{k}}{\partial x_{j}}\right) \\
& +2 v \frac{\partial \tau_{i k}}{\partial x_{j}} \frac{\partial^{2} \bar{u}_{i}}{\partial x_{j} \partial x_{k}}+2 v^{2} \tau\left(\frac{\partial^{2} u_{i}}{\partial x_{j} \partial x_{k}}, \frac{\partial^{2} u_{i}}{\partial x_{j} \partial x_{k}}\right)-2 v \tau\left(\frac{\partial u_{i}}{\partial x_{j}}, \frac{\partial F_{0 i}}{\partial x_{j}}\right)=0
\end{aligned}
$$

This equation is form-invariant under Euclidean transformations of the frame but is not frame-indifferent [5].

In the balance equation of the SGS kinetic energy (7) and in the balance equation of the SGS viscous dissipation (9) some tensors are unknown.

These unknown tensor are calculated by means of the closure relations.

These relations define the above mentioned unknown tensors as a function of known quantities by coefficients.

The calculation of these coefficients is carried out adopting the Germano's dynamic procedure.

\section{The spatially filtered concentration equation}

The filtered equation of the concentration of suspended solids is

$$
\frac{\partial \bar{C}}{\partial t}+\frac{\partial \bar{u}_{i} \bar{C}}{\partial x_{i}}+\frac{\partial}{\partial x_{i}}\left(\overline{u_{i} C}-\bar{u}_{i} \bar{C}\right)=0
$$

where $\bar{C}$ represents the spatially filtered concentration field.

The last term of equation (10) is a first-order tensor given by the generalised central moment relative to the velocity vector and the concentration and is defined as follows

$$
a_{i}=\tau\left(u_{i}, C\right)=\left(\overline{u_{i} C}-\bar{u}_{i} \bar{C}\right)
$$

The above tensor can be split in terms of generalised central moments

$$
a_{i}=L_{i}^{C}+C_{i}^{C}+R_{i}^{C}
$$

where

$$
\begin{gathered}
L_{i}^{C}=\tau\left(\bar{u}_{i}, \bar{C}\right)=\overline{\bar{u}_{i} \bar{C}}-\overline{\bar{u}_{i}} \overline{\bar{C}} \\
C_{i}^{C}=\tau\left(\bar{u}_{i}, C^{\prime}\right)+\tau\left(u_{i}^{\prime}, \bar{C}\right)=\left(\overline{\bar{u}_{i} C^{\prime}}-\overline{u_{i}} \overline{C^{\prime}}+\overline{u_{i}^{\prime} \bar{C}}-\overline{u^{\prime}} \overline{\bar{C}}\right) \\
R_{i}^{C}=\tau\left(u_{i}^{\prime}, C^{\prime}\right)=\overline{u_{i}^{\prime} C^{\prime}}-\overline{u_{i}^{\prime}} \overline{C^{\prime}}
\end{gathered}
$$


The sum of the second and third term on the right hand side of eqn (12) represents an unknown first- order tensor. In this paper a new closure relation for this unknown tensor is proposed.

The closure relation between this unresolved tensor and the gradient of the filtered concentration is defined by a second-order tensor which is assumed proportional to the subgrid turbulent velocity scale and the subgrid turbulence length scale:

$$
v_{i j}^{C}=c_{c} \sqrt{E} d_{i j}
$$

The above closure relation is:

$$
C_{i}^{c}+R_{i}^{c}=+\mathcal{C}_{c} \sqrt{E} d_{i n} \frac{\partial \bar{C}}{\partial x_{n}}
$$

Let $T\left(u_{i}, C\right)$ be the generalised central moment at the test level related to the velocity vector and the concentration, the following first-order tensor is defined

$$
A_{i}=T\left(u_{i}, C\right)=\widetilde{\widetilde{u_{i} C}}-\widetilde{\bar{u}_{i}} \widetilde{\bar{C}}
$$

where the symbol $\widetilde{(.)}$ indicates the filter operation at the test level.

In terms of generalised central moments $A_{i}$ can be split as:

$$
A_{i}=L_{i}^{C^{T}}+C_{i}^{C^{T}}+R_{i}^{C^{T}}
$$

where:

$$
\begin{aligned}
& L_{i}^{C^{T}}=T\left(\widetilde{\overline{u_{i}}}, \widetilde{\bar{C}}\right)=\widetilde{\widetilde{\overline{u_{i}}} \overline{\bar{C}}}-\stackrel{\widetilde{\overline{\underline{u}}}}{i} \widetilde{\overline{\bar{C}}}
\end{aligned}
$$

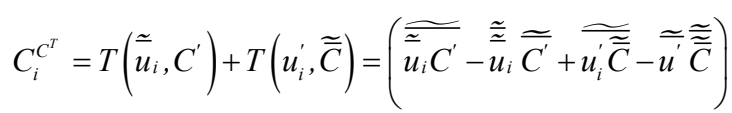

$$
\begin{aligned}
& R_{i}^{C^{T}}=T\left(u_{i}^{\prime}, C^{\prime}\right)=\widetilde{\widetilde{u_{i}^{\prime} C^{\prime}}}-\widetilde{\overline{u_{i}^{\prime}}} \widetilde{\bar{C}^{\prime}}
\end{aligned}
$$

The sum of the last two terms on the right hand side of eqn (19) is an unknown quantity that is modelled with an expression analogous to equation (17):

$$
C_{i}^{C^{T}}+R_{i}^{C^{T}}=c_{c} \sqrt{E^{T}} d_{i n}^{T} \frac{\partial \widetilde{\widetilde{C}}}{\partial x_{n}}
$$

The calculation of the coefficient $\mathrm{cc}$ is carried out by using the following identity:

$$
A_{i}-\widetilde{a_{i}}=\widetilde{\overline{u_{i}} \bar{C}}-\widetilde{\bar{u}_{i}} \widetilde{\bar{C}}
$$

Equation (24), with the use of the closure relations expressed by eqns (17) and (23), becomes 


$$
c_{r} \sqrt{E} d_{i n} \frac{\partial \bar{C}}{\partial x_{n}}-c_{r} \sqrt{E^{T}} d_{i n}^{T} \frac{\partial \widetilde{\bar{C}}}{\partial x_{n}}=\widetilde{\bar{u}_{i} \bar{C}}-\widetilde{\bar{u}_{i}} \widetilde{\bar{C}}-\widetilde{\widetilde{\widetilde{u}_{i}} \widetilde{\bar{C}}}+\widetilde{\widetilde{\bar{u}}_{i}} \widetilde{\widetilde{\bar{C}}}+\widetilde{\overline{\bar{u}_{i} \bar{C}}}-\widetilde{\overline{\bar{u}_{i}}} \overline{\bar{C}}
$$

where $E^{T}$ indicates the SGS kinetic energy relative to the test-filter and $d^{T}{ }_{\text {in }}$ indicates the second-order tensor associated with the subgrid turbulence length scales relative to the test-filter.

Equation (25) allows the dynamic calculation of the coefficient $c_{c}$.

The numerical integration of eqn (10) for the simulation of the filtered concentration field of suspended solids may be carried out once the boundary conditions have been defined.

The plane in proximity to the bottom, which defines the boundary for the concentration field, is placed immediately above the viscous sublayer, inside the buffer layer: the "Reference Concentration", $C_{r}$ is imposed on that plane as a boundary condition.

This reference concentration is calculated as function of the resolved tangential stress at the bottom, and the critical stress representing the threshold beyond which the movement of solid particles from the bottom is produced.

In particular, in the proposed model, the value of local and instantaneous reference concentration, $C_{r}$, is related to the resolved velocity field at the bottom by means of the formula proposed by Van Rijn [9]. The aforesaid Van Rijn's formula reads:

$$
C_{r}=0,015\left(\frac{D_{p}}{a}\right)\left(\frac{T^{1,5}}{D_{*}^{0,3}}\right)
$$

in which

a: distance from the bottom at which $C_{r}$ is calculated

$D_{p}$ : diameter of the solid particles

$$
D_{*}=D_{p}\left[\frac{(\delta-1) g}{v}\right]^{1 / 3}
$$

$\delta$ : relative density of the particle

$v$ : kinetic viscosity

$$
T=\frac{-2}{u_{*}^{2}-u_{*_{c r i t}}^{2}}
$$

and the quantity $\bar{u}^{2}$ which appears in eqn (28) is linked to the tangential stress at the bottom, $\tau_{\mathrm{f}}$, produced at each time step by the tangent resolved velocity component at the bottom, $\bar{u}$, by means of the following relation:

$$
\bar{u}^{2}=\frac{\tau_{f}}{\rho}=v \frac{\partial \bar{u}}{\partial y}
$$




\section{Results and discussion}

The proposed model is used to simulate the phenomenon of the re-suspension (from the bottom) of solid particles in a lid-driven cavity.

The values of the diameter and the relative density of the solid particles are, respectively, equal to $100 \mu \mathrm{m}$ and 1.65 .

The simulation of the lid driven cavity flow is performed at Reynolds number $R e=72,000$ which is based on the lid velocity $U_{\text {lid }}$ and the cavity length $B$. The $U_{\text {lid }}$ is directed towards the growing $x$ axis.

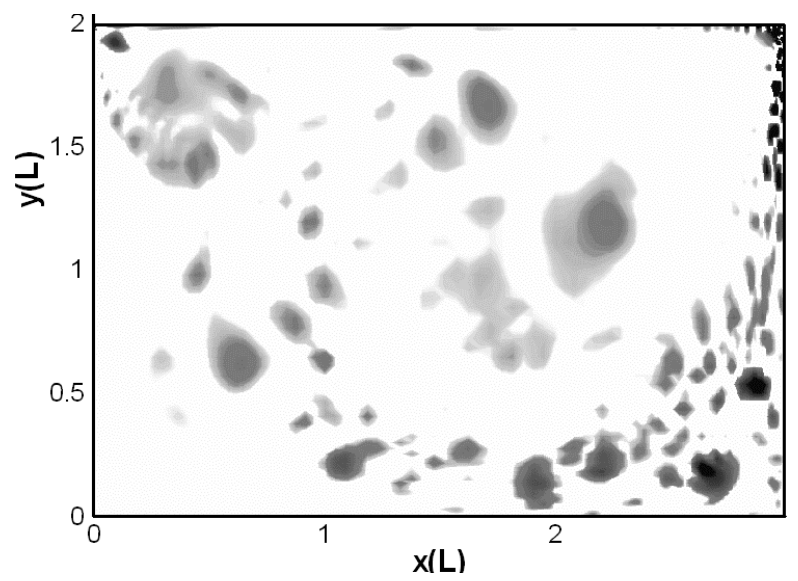

Figure 1: $\quad$ Vortex identification with $\lambda_{2}$ method, x-z plane.

In fig. 1 is shown, with the $\lambda_{2}$ visualisation method (Joeng \& Hussain, [8]), an instantaneous longitudinal section of the turbulent resolved velocity field inside the lid driven cavity at $R e=72,000$. The near wall vortex structures (inside the turbulent boundary layer) are clearly identified: the dimensions of the spatial discretisation steps allow the optimal simulation of the above mentioned vortex structures that govern the transport, the production and the dissipation of the turbulent kinetic energy.

As can be seen in fig. 1, starting from the top right hand corner and going towards the bottom, vortex structures of small dimensions following the main flow (which moves in a clockwise direction) are formed along the right vertical wall and grow in dimension. In correspondence with the abscissa $\mathrm{x}=2$, the main clockwise moving vortex structure is at a minimum distance from the bottom, and from there it moves away transporting towards the centre of the domain the small secondary vortexes that form on the bottom. At the same time, in proximity to the bottom right corner, a vortex structure (that moves in a clockwise direction and is confined to the area between the main clockwise vortex and the walls) is formed and destroyed.

In fig. 2-5 is shown a sequence of longitudinal sections of instantaneous concentration fields inside the lid driven cavity produced by a re-suspension 
from the bottom of the solid particles caused by a turbulent flow characterised by $R e=72,000$. In this case the maximum tangential stresses at the bottom are produced in correspondence with the clockwise moving vortex structure and with the appearance of the anti-clockwise vortex structure confined to the bottom right hand corner. The particles, that are re-suspended from the bottom in correspondence with $\mathrm{x}<2$, are transported upwards by the main clockwise vortex rapidly scattered in the whole calculation domain; the particles, that are resuspended from the bottom in correspondence with $x>2$, are caught by the anticlockwise vortex structure confined to the bottom right hand corner where they tend to precipitate.

The visualized concentration values, expressed as a percentage of volume fraction, are inclusive among $1 \times 10^{-4}$ and $2.4 \times 10^{-1}$.

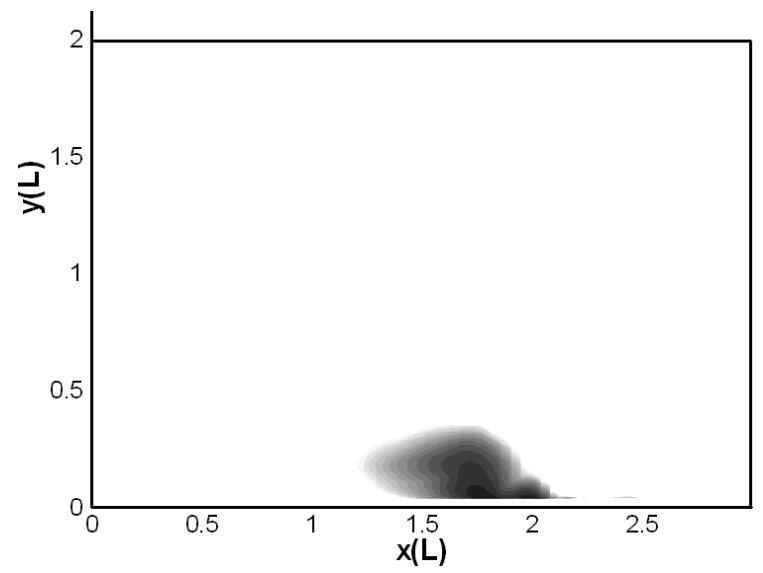

Figure 2: $\quad$ Lid Driven Cavity: filtered concentration field.

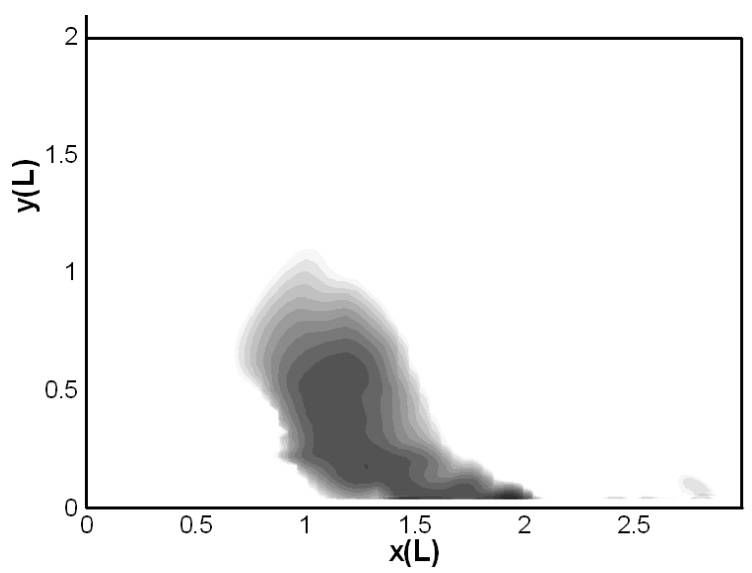

Figure 3: Lid Driven Cavity: filtered concentration field. 


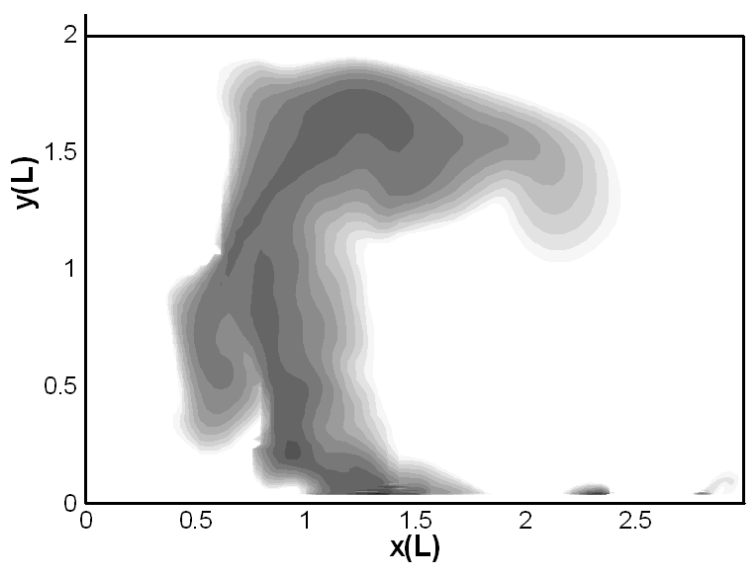

Figure 4: $\quad$ Lid Driven Cavity: filtered concentration field.

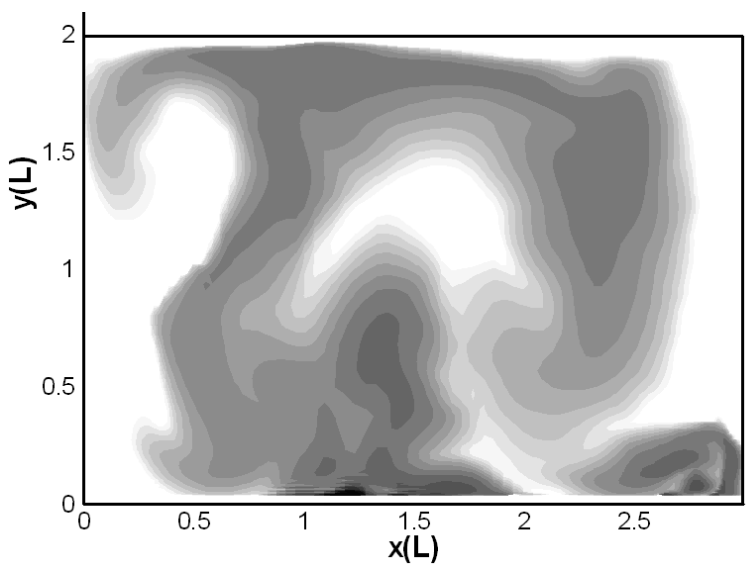

Figure 5: Lid Driven Cavity: filtered concentration field.

\section{Conclusions}

In this paper a new LES model is presented together with a new methodology for the simulation of concentration fields of solids in suspension which is coherent with the LES methodology. The proposed closure relation for the generalized SGS turbulent stress tensor: a) complies with the principle of turbulent frame indifference; b) takes into account both the anisotropy of the turbulence velocity scales and turbulence length scales. The concentration field is simulated by the spatially filtered equation of the concentration. In this equation the first order tensor (produced by the correlation between velocity and concentration) is related to the gradient of the concentrations resolved according to an original dynamic Germano procedure.

By the proposed model the phenomenon of re-suspension, from the bottom, of solid particles in a lid driven cavity at $R e=72.000$ is simulated. 


\section{References}

[1] Hutter, K, Jonk, K, Coontinum methods of Physical of Modelling, Springer, 2004.

[2] Germano M, Piomelli U, Moin P, Cabot WH, A dynamic subgrid scale eddy viscosity model. Phys. Fluids A3, 1760-1765, 1991.

[3] Meneveau C, Lund TS, Cabot WH, A Lagrangian dynamic subgrid-scale model of turbulence, J. Fluid Mech. 319, 353-385.

[4] Ghosal S, Lund TS, Moin P, Aksevoll K, A dynamic localisation model for large-eddy simulation of turbulent flows, J. Fluid Mech. 286, 229-255, 1995.

[5] Gallerano F., Pasero E., Cannata G., A dynamic two-equation Sub Grid Scale model. Continuum Mech. Thermodyn. 17, 101-123, 2005.

[6] Chong, M.S. Perry, A.E. Cantwell, B.J, A general classification of three dimensional flow field, Phys. Fluids. A 2, 765, 1990.

[7] Hunt. J.C.R., Vassilicos, J.C. \& Kevlahan, N.K.R., 'Turbulence-. A state of nature or a collection of phenomena?', in Branover, H. \& Unger, Y. (Eds) Progress in turbulence Research, 7th Beer Sheva Int. Sem. on MHD flows and turbulence, Beer eb. 1993, Progress in Astronautics Series, AIAA Sheva, Israel, Feb. 1993.

[8] Joeng, J. Hussain, F, On the identification of a vortex, J.Fluid Mech. 285, 69-94, 1995.

[9] Van Rijn L.C., Sediment transport, Delft Hydraulic Laborator. Vol. 334, 1985. 